\title{
AN APPROACH FOR TESTING REAL TIME PROTOCOL ENTITIES
}

\author{
Ahmed Khoumsi \\ Université de Sherbrooke, Dep. GEGI, Sherbrooke, CANADA \\ khoumsi@gel.usherb.ca
}

Mehdi Akalay, Rachida Dssouli, Abdeslam En-Nouaary

Université de Montréal, Dep. IRO, Montréal, CANADA

(akalay,dssouli,ennouaar)@iro.umontreal.ca

\section{Louis Granger}

École Polytechnique de Montréal, Dep. GEGI, Montréal, CANADA

louis.granger@mail.polymtl.ca

\begin{abstract}
We propose a two-step method and an architecture for testing real-time protocol entities. In the first step, the timed specification of the implementation under test is transformed into an equivalent untimed specification. Then in the second step, test methods for non-real-time systems may be used. In comparison with other test methods using similar approaches, our method avoids state explosion. A transformation algorithm of the first step and a test architecture are proposed.
\end{abstract}

Keywords: Test generation, Test architecture, Implementation under test, Real time protocol entity, Timed automata, Discrete time, Minimization.

\section{INTRODUCTION}

Many formal methods are used to describe timed systems; real-time logic, timed Petri nets, and timed automata are among the most popular. Two approaches are known to represent the time : continuous time [2] and discrete time $[15,10]$. With continuous time, time measures represent exact values of time. With discrete time, a unit of clock time (uct) is defined and time measures are integer values which are incremented after the passing of each uct. Various 
techniques and tools have been developed to ensure formal verification of timed systems, such as COSPAN, KRONOS, UPAAL and HYTECH. But few work has been done for testing timed systems; for example :

- In [12] timers and counters are used to guarantee a reliable transmission of messages in a given bounded time. The used model allows to describe constraints on delays separating sends and corresponding receives.

- In [14] temporal logic formulas are extended with discrete time. Tests are generated from formulas written in that logic. Their method allows to describe simple formulas using a single variable.

- In [3] the authors generate tests from a Constraint Graph (CG). Their model allows to describe constraints on delays separating consecutive events.

- In [5] the authors propose a test method based on the transition tour of the entire region graph of the control part. A state is identified only by a single empirical value for each clock. The fault coverage of the method is limited.

- In [17] the authors propose a theoretical method for test cases generation based on a variant of the Timed Automata of [2]. This is the first approach inspired by methods for untimed systems.

- In [6] the authors continue in the same direction as [17] and provide a practical and complete method for test cases generation.

In this article ${ }^{1}$, we propose a test method and a test architecture for timed systems. The proposed test method consists of two steps : (1) the timed specification of the $\mathcal{I U T}$ (Implementation Under Test) is transformed into an equivalent untimed specification; and (2) existing test methods for untimed systems may then be used to generate test sequences. Our work is inspired from [6] which uses a similar two-step approach. An important difference with [6] is that we use discrete time while in [6] continuous time is used. Discrete time does not allow to represent exactly the physical behaviour of the system. However, discrete time is sufficiently accurate in many instances, for instance when dealing with digital control systems [11].

In [6] after the transformation of the first step, the elapsing of each (uct) of each clock is represented by a transition $\nu$. Therefore a state explosion problem arises. Our method avoids state explosion by representing only "relevant" time elapsing. For instance, if the possible behaviour of a system changes only after ten ucts, then only the elapsing of the tenth uct will be represented. Although this optimisation is inspired from [1] which uses a continuous time, it is of no use to [6]. In fact, in continuous time the relevant time elapsing cannot in

${ }^{1}[9]$ is a longer version with more details 
general be related to any concrete aspect, while in discrete time the relevant time elapsing can be related to the expiration of a real timer. We propose an algorithm to realize the transformation of the first step. We also propose a test architecture applicable to the specifications obtained at the first step. Henceforth FSA denotes "Finite State Automaton".

The remaining of this article is structured as follows. Sect. 2 describes the model of timed automata (TA) and its corresponding fault model. In Sect. 3 we present our tick-FSA model and its minimization. In Sect. 4 we propose the se-FSA model which, in comparison to tick-FSA, avoids state explosion. And then we present an algorithm for transforming a TA into a se-FSA. Sect. 5 presents a test architecture applicable to specifications described by se-FSAs. In Sect. 6 we show how test cases are generated. And finally in Sect. 7 we conclude by discussing some future work.

\section{TIMED AUTOMATA AND FAULT MODEL \\ 2.1. Model of Time and Timers}

We consider a digital clock [10] which generates a tick at a constant frequency; the delay between two consecutive ticks is called unit of clock time (uct). The time is modeled by a variable $\tau$ which is initially equal to zero and is incremented by one after the passing of each uct (Fig. 1.a). With such a model, there is an inaccuracy of one uct on the instants of events and an inaccuracy of two ucts on the delays separating two events [10] (Fig. 1.b). A timer is an integer variable which : (1) is automatically incremented after each tick and (2) may be set to zero at the occurrence of every event.

\begin{tabular}{|c|c|c|c|c|c|c|c|c|c|c|c|c|c|}
\hline & & & & & & & Values of $\tau$ & 0 & 1 & 2 & 3 & 4 & $\ldots$ \\
\hline$\frac{\text { Values of } \tau}{\text { tick }}$ & O & $\frac{1}{1}$ & 2 & 3 & 4 & $\underset{\text { Time axis }}{\ldots .}$ & tick & (b) & & & $T$ & 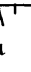 & Time axis \\
\hline
\end{tabular}

Figure 1. (a) Time model; (b) Accuracy of the time model

\subsection{Timed Automata (TA)}

We consider a set of timers $\mathcal{T}=\left\{t_{1}, \cdots, t_{N_{t}}\right\}$, and we define a canonical Enabling Condition (EC) as being any formula in the form " $t_{i} \sim k$ ", where $\sim \in\{\langle\rangle,, \leq, \geq,=\}$. More generally, a EC may consist of a single canonical EC or of a conjunction of canonical ECs. A EC may also be the constant True. We also define $\mathcal{E C}_{\mathcal{T}}$ as being the set of ECs depending on timers of $\mathcal{T}$, and $\mathcal{P}_{\mathcal{T}}$ as being the set of subsets of $\mathcal{T}$.

A TA may be obtained from a FSA if we associate an EC and a set $\mathrm{Z}$ of timers to each transition $\operatorname{Tr}$ of the FSA. More formally, a TA may be defined by $\left(\mathcal{L}, \mathcal{E}, \mathcal{T}, \mathcal{T} r, l_{0}\right)[10]$ where : $\mathcal{L}$ is a finite set of locations, $l_{0}$ is the initial location, $\mathcal{E}$ is a finite set of events, $\mathcal{T}$ is a finite set of timers, and 
$\mathcal{T} r \subseteq \mathcal{L} \times \mathcal{E} \times \mathcal{L} \times \mathcal{E C}_{\mathcal{T}} \times \mathcal{P}_{\mathcal{T}}$ is a transition relation. A transition is therefore defined by $\operatorname{Tr}=\langle q ; \sigma ; r ; E C ; Z\rangle$ where : $q$ and $r$ are origin and destination locations, $\sigma$ is the event of the transition, Tr may occur only if $E C=t r u e$, and after the occurrence of $\operatorname{Tr}$ the timers in $Z$ are set to zero. $Z$ is called reset of $\operatorname{Tr}$.

A TA allows to express constraints on the number of ticks between events. For example, to specify that there may be 1 to 3 ticks between transitions $\operatorname{Tr} 1$ and $\operatorname{Tr} 2$, we may use a timer $t_{1}$ as follows : the $Z$ of $\operatorname{Tr} 1$ is $\left\{t_{1}\right\}$ and the $E C$ of $\operatorname{Tr} 2$ is $\left(t_{1} \geq 1\right) \wedge\left(t_{1} \leq 3\right)$. Note that a state $s$ of a system specified by a TA may be defined by $(l, t)$, where $l$ is a location and $t$ is an $N_{t}$-tuple specifying the current value of each timer.

\subsection{Example of TA}

We consider the simplified version of the sender of the CSMA/CD communication protocol [19] which is described by the TA of Fig. 2 where : (1) $\lambda$ is the transmission time of a message and $\sigma$ is the worst case propagation delay; (2) a single timer $t$ is used; (3) ? $u$ (resp. ! $u$ ) means " $u$ is received (resp. sent)"; and (4) a transition $\operatorname{Tr}=\langle q ; \sigma ; r ; E C ;\{t\}\rangle$ is labelled by " $E C ; \sigma ; t:=0$ ". The absence of EC or of timers to reset are indicated by “-". As described in Fig. 2 :

- The sender is initially at location idle where it waits for a message to send. When the latter is received (?send), the sender reaches location ready.

- When location ready is reached, the sender verifies the bus. If the latter is free (?free), the sender starts the transmission (!begin) and reaches location transmit; otherwise the sender reaches location retry either if the bus is busy (?busy) or if a collision is detected (?cd).

- When location retry is reached, the sender waits for the availability of the bus during twice the worst propagation delay $(t=2 \sigma)$ before reattempting the transmission of the message (!begin) and then reaching location transmit. If a collision is detected in location retry before the delay $2 \sigma(t<2 \sigma)$, then the sender begins again to wait for the availability of the bus (selfloop).

- When location transmit is reached (from ready or retry), the transmission is started (!begin). If a collision is detected (?cd) before the worst propagation delay $(t<\sigma)$, the sender goes to location retry; otherwise it terminates sending the message (!end) after exactly $\lambda$ ucts $(t=\lambda)$ and goes to location idle. 


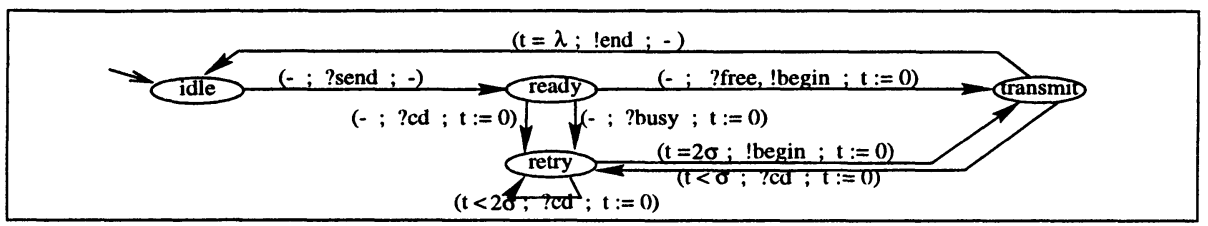

Figure 2. CSMA-CD sender

\subsection{Fault Model}

Let SP and IUT be two TAs describing a specification and its implementation, respectively. We may categorize faults that may arise in an implementation of a timed system by : (1) faults independent on timing constraints; and (2) timing faults [7]. For the first category, we consider the types of faults given in [13] : output faults, transfer faults and hybrid faults. The second category of faults are caused by the non respect by IUT of timing constraints associated to outputs of SP. During a testing process and for a given test sequence, the tester respects timing constraints of inputs and checks whether timing constraints of outputs are respected. An essential type of timing faults may be defined as follows : (1) SP requires that the $\mathcal{I U T}$ sends an output $\sigma$ at an instant $t \in[t 1, t 2]$; and (2) IUT allows the sending of $\sigma$ at certain instants which do not fall within $[t 1, t 2]$. In reality, with discrete time $t, t 1$ and $t 2$ are numbers of ticks. For example, in the specification of Fig. 2, when location transmit is reached, the output !end must occur after $\lambda$ ticks. The $\mathcal{I U} \mathcal{T}$ is faulty if !end occurs before the $\lambda$ th tick or after the $(\lambda+1)$ th tick.

\section{TICK-FSAS AND THEIR MINIMIZATION}

We show here how a TA may be transformed into an equivalent FSA called tick-FSA. Then we show in an example how state explosion may be avoided by combining states of the tick-FSA.

\subsection{Transforming TA into Tick-FSA}

A TA may be transformed into an equivalent FSA called tick-FSA where the event tick is represented by a transition [10]. A state of a tick-FSA may be defined by $(l, t)$ where : (1) $l$ is a location of the corresponding TA and (2) $t$ is a $N_{t}$-tuple specifying the current value of each timer $\left(N_{t}\right.$ is the number of timers). The transformation from a TA into a tick-FSA can lead to a state explosion problem [10].

We consider the example of Fig. 2 where : the bus is $2^{20}$ bits/second, the propagation delay $\sigma$ is 25 ucts, the length of each message is $2^{10}$ bytes, and 1 second $=10^{6}$ ucts. We compute that the delay $\lambda$ to transmit a message, including the propagation delay, is 806.25 ucts. Therefore, events !begin and 
!end are separated by 806 ticks. The resulting tick-FSA which illustrates these results is represented on Fig. 3.

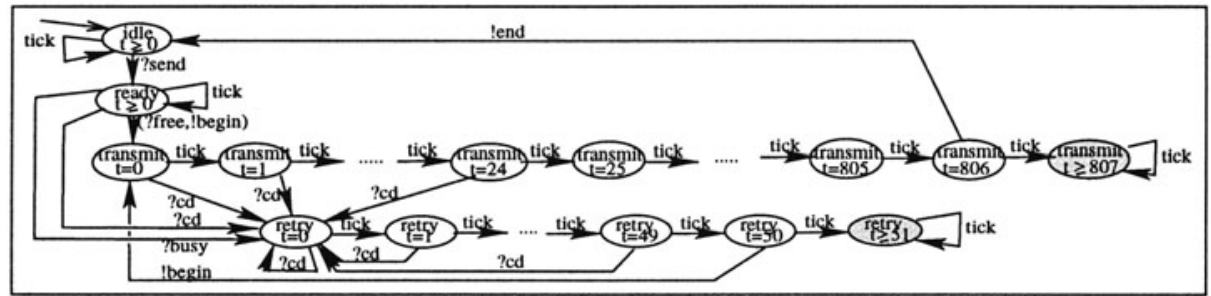

Figure 3. State explosion with a tick-FSA

\subsection{Combining States of a Tick-FSA}

For example, the 808 states of the tick-FSA of Fig. 3 which are associated to location transmit can be combined into :

Group 1: transmit, $t \leq 24$ : from which ?cd leads to (retry, $t=0$ ), Group 2 : transmit, $25 \leq t \leq 805$ : from which no event $(\neq t i c k)$ occurs, Group 3: transmit, $t=806$ : from which !end leads to (idle, $t \geq 0$ ), Group $4:$ transmit, $t \geq 807$ : which is a deadlock.

Transitions between these groups are :

(Group $1 \rightarrow$ Group 2) is the 25th tick from (transmit, $t=0$ ),

(Group $2 \rightarrow$ Group 3 ) is the 806th tick from (transmit, $t=0$ ),

(Group $3 \rightarrow$ Group 4 ) is the 807 th tick from (transmit, $t=0$ ).

These combinations illustrate the possibility to avoid state explosion. In Sect. 4 we propose the se-FSA model which is the result of this kind of combinations, and we present an algorithm for transforming a TA into a se-FSA without using an intermediate tick-FSA.

\section{TRANSFORMING TA INTO A SE-FSA}

\subsection{Two New Types of Events}

? Set $\left(t_{i}, k\right)$ means : timer $t_{i}$ is set to zero and will expire when its value is equal to $k$. ? Set $\left(t_{i}, k_{1}, k_{2}, \cdots, k_{p}\right)$ means that $t_{i}$ is set to zero and will expire several times when its value is equal to $k_{1}, k_{2}, \cdots, k_{p}$.

$! \operatorname{Exp}\left(t_{i}, k\right)$ means : timer $t_{i}$ expires and its currewnt value is $k$.

Therefore, each event ? $\operatorname{Set}\left(t_{i}, k\right)$ is followed (after a delay $k$ ) by $! \operatorname{Exp}\left(t_{i}, k\right)$, and each event ?Set $\left(t_{i}, k_{1}, k_{2}, \cdots, k_{p}\right)$ is followed by the sequence : $! \operatorname{Exp}\left(t_{i}, k_{1}\right), ! \operatorname{Exp}\left(t_{i}, k_{2}\right), \cdots, ! \operatorname{Exp}\left(t_{i}, k_{p}\right)$. An event $\operatorname{Set}$ (resp. Exp) is associated with Sign “?” (resp. "!") because, as we will see in Sect. 5, Set (resp.Exp) is sent (resp. received) by the tester and may therefore be conceptually considered as an input (resp. output) of the system under test $(\mathcal{S U T})$. 
In [4], events Set and Exp have been used differently than here, for verifying timed systems.

\subsection{Transformation Algorithm}

We propose here an algorithm for transforming a TA into a FSA with events Set and Exp, which we denote se-FSA. The algorithm consists of four steps which may be intuitively introduced as follows :

Step 1 : Rewrite the ECs by using only " $<$ " and " $\geq$ ". This rewriting is convenient because the value (true or false) of a canonical EC " $t<k$ " or " $t \geq k$ " changes once exactly when $t=k$. After the rewriting, the only relevant information for checking " $t \sim k$ ", will be : (1) whether $t$ has been set to zero and (2) whether its value has reached $k$.

Step 2 : Associate an event ? Set $(t, k)$ to each transition which : (1) resets a timer $t$ and (2) is followed (not necessarily in the next transition) by an $\mathrm{EC}$ " $t \sim k$ ".

Step 3 : For each location $L$ :

1 Associate an event $! \operatorname{Exp}(t, k)$ to each " $t \sim k$ " which is in an outgoing transition of $L$.

2 Construct all the sequences consisting of all the determined events Exp. Each of these sequences, which we denote Exp-sequence, corresponds to an order of events Exp.

3 Remove certain impossible Exp-sequences by using certain rules. For example, let $! \operatorname{Exp}\left(t 1, k_{1}\right)$ and $! \operatorname{Exp}\left(t 2, k_{2}\right)$ be contained in the set of constructed Exp-sequences, where $k_{1} \leq k_{2}$. If in all paths which allow to reach location $L, ? \operatorname{Set}\left(t 1, k_{1}\right)$ is before $? \operatorname{Set}\left(t 2, k_{2}\right)$, then any Exp-sequence where $! \operatorname{Exp}\left(t 1, k_{1}\right)$ is after $! \operatorname{Exp}\left(t 2, k_{2}\right)$ is removed.

4 Simplify each constructed Exp-sequence $S e q$ by removing certain "irrelevant" events $E x p$ according to the following rule. If just after an event Exp the EC of a transition $\operatorname{Tr}$ becomes false, then remove all the following events Exp which are associated exclusively to ECs of $T r$. The removed events are irrelevant because when a transition becomes false, it remains false in the whole remaining part of Seq. This simplification will be illustrated in the example of Fig. 6.

5 For each constructed Exp-sequence, we construct a sequence of states $L_{1}, L_{2}, \cdots$ which are connected by this Exp-sequence.

6 For each outgoing transition $\operatorname{Tr}$ of $L$ leading to any $L^{\prime}$, we construct a transition executing the same event than $\operatorname{Tr}$ from every $L, L_{1}, L_{2}, \cdots$ where the EC of $\operatorname{Tr}$ is true. All the constructed transitions lead to $L^{\prime}$. 
Step 4 : The obtained FSA is determinized and minimized, and undesirable states are removed.

For the TA of Fig. 2 the result just before the removal of undesirable states is represented in Fig. 4. Here is an intuitive explanation of how this se-FSA is constructed : $(\lambda=806$ and $\sigma=25)$

1 “ $t=806$ " is rewritten " $(t \geq 806) \wedge(t<807)$ ", and " $t=50$ " is rewritten " $(t \geq 50) \wedge(t<51)$ ".

2 Events Set are determined as follows:

- " $t:=0$ " of the two transitions leading to transmit are followed by comparisons :

" $t<25$ " of the transition transmit $\rightarrow$ retry, and

“ $(t \geq 806) \wedge(t<807)$ " of the transition transmit $\rightarrow$ idle.

Therefore ?Set $(t, 25,806,807)$ is associated to the transitions leading to transmit.

- " $t:=0$ " of the four transitions leading to retry are followed by comparisons :

" $t<50$ " of the transition retry $\rightarrow$ retry, and

" $(t \geq 50) \wedge(t<51)$ " of the transition retry $\rightarrow$ transmit.

Therefore ? $\operatorname{Set}(t, 50,51)$ is associated to the transitions leading to retry.

3 Events $E x p$ are constructed as follows :

- Three events ! $\operatorname{Exp}(t, 25), ! \operatorname{Exp}(t, 806)$ and $! \operatorname{Exp}(t, 807)$ are consequences of ? $\operatorname{Set}(t, 25,806,807)$.

- Two events $! \operatorname{Exp}(t, 50)$ and $! \operatorname{Exp}(t, 51)$ are consequences of $? \operatorname{Set}(t, 50,51)$.

- The sequence $(! \operatorname{Exp}(t, 25), ! \operatorname{Exp}(t, 806), ! \operatorname{Exp}(t, 807))$ "divides" transmit into four states which correspond to the four groups introduced in Sect. 3.2.

- The sequence $(! \operatorname{Exp}(t, 50), ! \operatorname{Exp}(t, 51))$ divides retry into three states : $($ retry,$t \leq 49),($ retry,$t=50)$ and $($ retry,$t \geq 51)$.

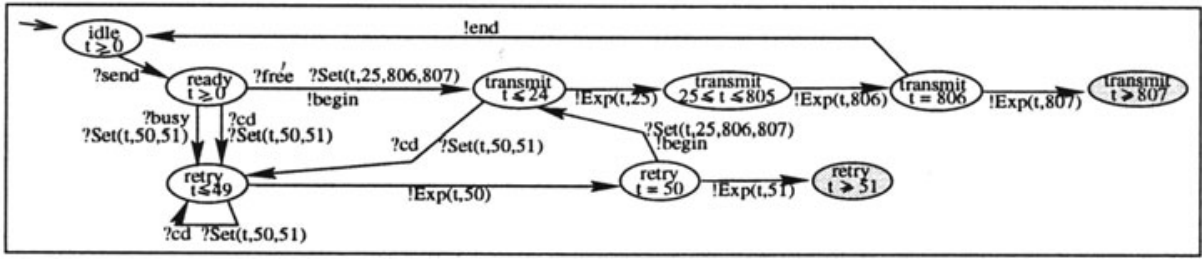

Figure 4. se-FSA obtained from the TA of Fig. 2. Undesirable states are marked. 


\subsection{Operational Presentation of the Algorithm}

\begin{tabular}{|c|c|}
\hline Step 1 & $\begin{array}{l}\text { For every canonical EC: } \\
\text { If the EC is " } t_{i}=k \text { " then rewrite it into " }\left(t_{i} \geq k\right) \wedge\left(t_{i}<k+1\right) \text { ", } \\
\text { If the EC is " } t_{i} \leq k \text { " then rewrite it into " } t_{i}<k+1 \text { ", } \\
\text { If the EC is " } t_{i}>k \text { " then rewrite it into " } t_{i} \geq k+1 \text { ". } \\
\text { End }\end{array}$ \\
\hline Step 2 & $\begin{array}{l}\text { For every location } L \text { of the TA : } \\
\text { For every outgoing transition } \operatorname{Tr} \text { of } L: \\
\text { For every timer } t \text { which is reset by } \operatorname{Tr} \text { : } \\
\text { Search ECs in transitions which are reachable from } \operatorname{Tr} \text { without } \\
\text { crossing a transition which resets } t \\
\text { For every EC " } t \sim k^{\prime} \text { which has been found : } \\
\text { Associate the event ? Set }(t, k) \text { to } T r \\
\text { End } \\
\text { End } \\
\text { Events? } \operatorname{Set}\left(t, k_{1}\right), ? \operatorname{Set}\left(t, k_{2}\right), \cdots, ? \operatorname{Set}\left(t, k_{p}\right) \text { may be replaced by } \\
\text { ? } \operatorname{Set}\left(t, k_{1}, k_{2}, \cdots, k_{p}\right) \\
\text { End } \\
\text { End }\end{array}$ \\
\hline Step 3 & 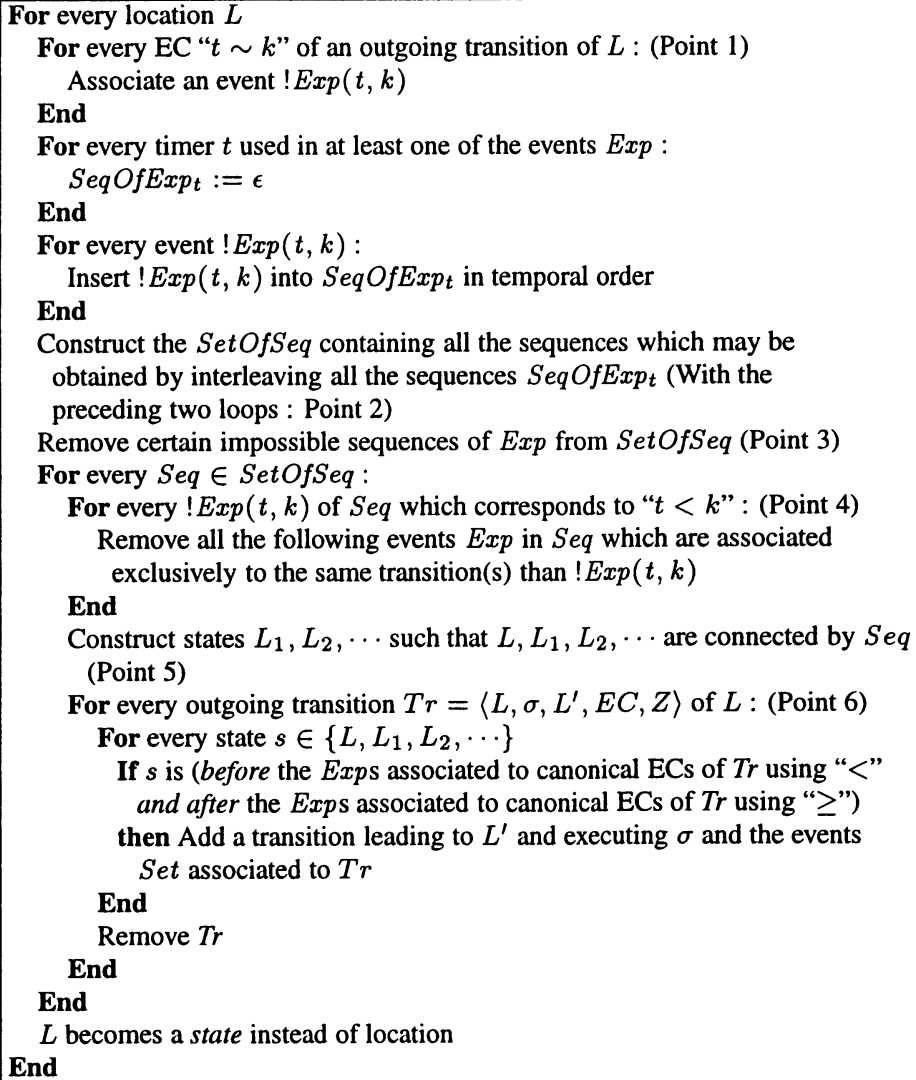 \\
\hline Step 4 & $\begin{array}{l}\text { Determinize and minimize the obtained FSA } \\
\text { Remove the undesirable states }\end{array}$ \\
\hline
\end{tabular}


with the the TA of Fig. 5 which uses timers $t 1$ and $t 2$. The results of the four steps are represented in Fig. 6. In Fig. 6.c (resp. d) we show the result of Step 4 without (resp. with) the simplification. The latter may be explained as follows. The transition $\operatorname{Tr}$ executing $\phi$ has the EC " $(t 1<4) \wedge(t 2<3)$ ", and therefore it becomes disabled after the first of $! \operatorname{Exp}(t 1,4)$ and $! \operatorname{Exp}(t 2,3)$. For this reason, it is useless to represent the second of the two Exp. This implies that every simplified sequence contains either $! \operatorname{Exp}(t 1,4)$ or $! \operatorname{Exp}(t 2,3)$.

In general a timer $t$ may expire at an instant $\tau$ and its expiration is either : (1) immediately relevant; or (2) irrelevant and will become relevant at a future instant $\nu(\nu>\tau)$; or (3) irrelevant and will never become relevant. Case (3) can be considered as a particular case of Case (2) when $\nu=\infty$. In our transformation algorithm, in Cases (2) and (3) we have conceptually delayed the expiration until instant $\nu$. As an illustration of Case (3) in the example of Fig. 6, when $\phi$ occurs in State $L 2_{0}$ and then leads to State $L 0_{0}$, none of the events $! \operatorname{Exp}\left(t_{1}, 2\right), ! \operatorname{Exp}\left(t_{1}, 4\right), ! \operatorname{Exp}\left(t_{2}, 3\right)$ and $! \operatorname{Exp}\left(t_{2}, 5\right)$ is relevant in State $L 0_{0}$. For this reason, although their occurrences remain possible in $L 0_{0}$, these events have been conceptually delayed to the instant $\nu=\infty$. In Sect. 5.1.3, we will see how this delaying action can be realized physically.

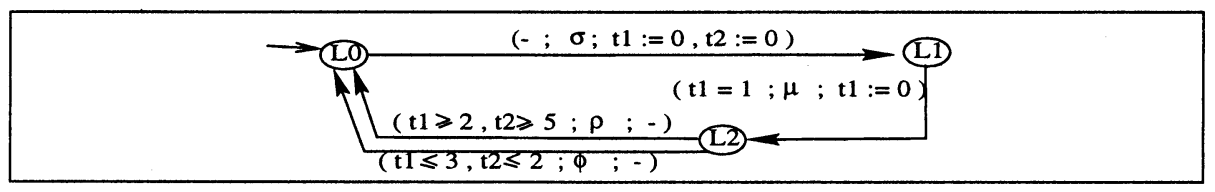

Figure 5. Example of TA using two timers

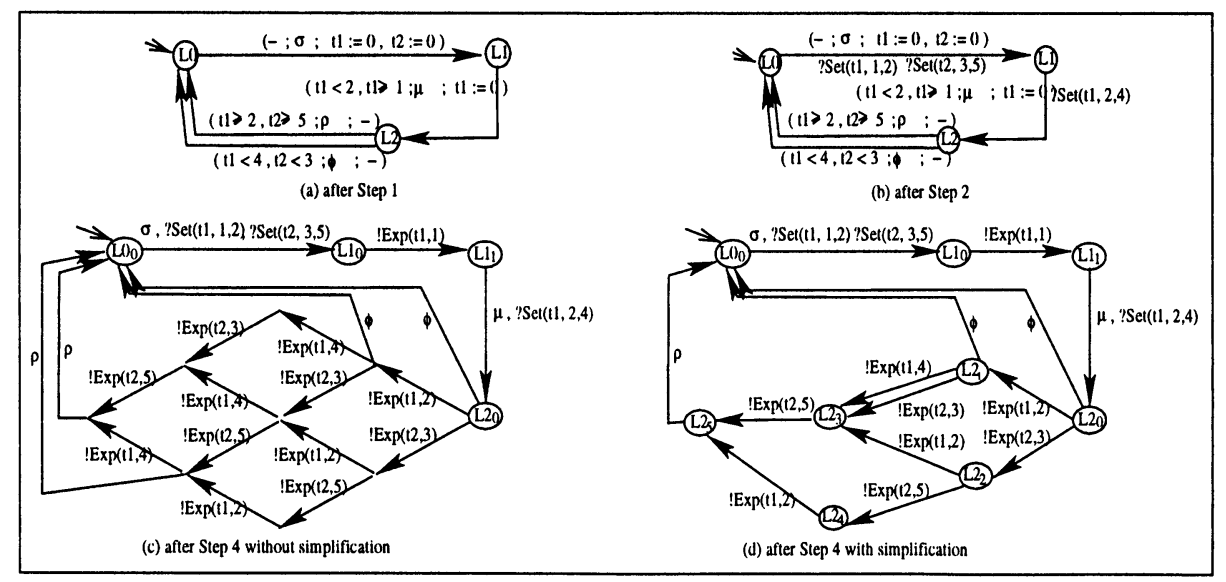

Figure 6. Results of the algorithm when it is applied to the TA of Fig. 5 


\section{TEST ARCHITECTURE}

We propose a test system $(\mathcal{T S})$ consisting of three modules called TestController, Exp-Delayer and Timer-Handler, respectively. The interest of such a $\mathcal{T S}$ is that it guarantees the following equivalence.

TESTING EQUIVALENCE : Let $\mathrm{S} 1$ be a TA and $\mathrm{S} 2$ be the se-FSA obtained from $S 1$ using the algorithm of Sect. 4 . The following two points are equivalent :

1 The $\mathcal{I U T}$ is conformant to the specification $\mathrm{S} 1$.

2 The $\mathcal{S U} \mathcal{T}$ (System Under Test) consisting of the set $\{\mathcal{I} \mathcal{U} \mathcal{T}$, Exp-Delayer, Timer-Handler $\}$ is conformant to the specification $S 2$.

\subsection{Test System}

The test system $(\mathcal{T S})$ consists of three modules : Test-Controller, TimerHandler and Exp-Delayer.

5.1.1 Test-Controller. This module executes test sequences generated from the se-FSA generated itself from the TA describing the specification. Therefore Test-Controller : (1) sends inputs of the $\mathcal{I U T}$ and events Set; and (2) receives outputs of the $\mathcal{I U} \mathcal{T}$ and events Exp.

5.1.2 Timer-Handler. This module consists of : (1) a process $P C$ which generates the event tick at a constant frequency $=\frac{1}{u c t}$; and (2) a process $P T_{i}(k)$ created with the reception of each ? Set $\left(t_{i}, k\right) . k$ ucts after its creation, $P T_{i}(k)$ generates $! \operatorname{Exp}\left(t_{i}, k\right)$ and then terminates. If a ? $\operatorname{Set}\left(t_{i}, p\right)$ is generated before the termination of $P T_{i}(k)$, then the latter is killed and a new process $P T_{i}(p)$ is created.

5.1.3 Exp-Delayer. This module realizes the delaying action of events Exp which has been introduced at the end of Sect. 4. To achieve this delaying, Exp-Delayer :

1 "shows" the occurrence of an event $\operatorname{Exp}(t, k)$ if the latter is allowed;

2 "hides and stores" the occurrence of an $\operatorname{Exp}(t, k)$ if the latter is not allowed;

3 "removes" a stored event $\operatorname{Exp}(t, k)$ when a $\operatorname{Set}(t, *)$ is executed;

4 "generates" automatically and immediately a (non-removed) stored $\operatorname{Exp}(t, k)$ when the latter becomes allowed.

For choosing between "shows", "hides and stores" and "generates", the ExpDelayer needs to know the current state of the se-FSA. Therefore it needs to observe all the events (inputs, outputs, Set and Exp). For performing the 
"removes" action, the Exp-Delayer needs to observe Set events. A specification of the Exp-Delayer may therefore be obtained by modifying the se-FSA as follows :

- All events become inputs (Exp-Delayer observes all the events).

- Every input $\operatorname{Exp}(t, k)$ is replaced by :

“? $\operatorname{Exp}(t, k), ! \operatorname{Exp}(t, k)$ " if $\operatorname{Exp}(t, k)$ is not stored (Exp-Delayer "shows" the input ? $\operatorname{Exp}(t, k))$,

"! $\operatorname{Exp}(t, k)$ " if $\operatorname{Exp}(t, k)$ is stored (Exp-Delayer "generates" the stored $\operatorname{Exp}(t, k))$.

- In every state $q$, add selfloops of inputs Exps which are not allowed in $q$ and associate to them the action "hides and stores".

- To every input ? $\operatorname{Set}(t, k)$, associate the action "remove $(! \operatorname{Exp}(t, k))$ ".

5.1.4 Test system $(\mathcal{T S})$ and System Under Test $(\mathcal{S U T})$. Our test system $(\mathcal{T S})$ has therefore the structure represented in Fig. 7. Let $\mathrm{S} 1$ be a TA describing the specification and $\mathrm{S} 2$ be the se-FSA obtained from S1. The proposed structure allows to test the conformance of the set $\{\mathcal{I U} \mathcal{T}$, TimerHandler, Exp-Delayer $\}$ to the specification S2. From the testing equivalence (see beginning of Sect. 5), we deduce that this structure allows to test the conformance of the $\mathcal{I U T}$ to the specification $\mathrm{S} 1$. Testing equivalence can be intuitively explained as follows : the Timer-Handler and the Exp-Delayer are assumed correct, and therefore the detection of any error by the Test-Controller in the set $\{\mathcal{I U} \mathcal{T}$, Timer-Handler, Exp-Delayer $\}$ implies that the $\mathcal{I U} \mathcal{T}$ is faulty.

Note that certain transitions of the se-FSA may have two inputs : an event Set and an input for the $\mathcal{I U T}$ (see Fig. 4). This is not a problem because the two inputs are sent by the Test-Controller to different destinations $(\mathcal{I U T}$ versus $\{$ Timer-Handler, Exp-Delayer $\}$. Note that the action "generates" of the Exp-Delayer must be immediate because the testing equivalence is guaranteed if and only if no event of the $\mathcal{I U T}$ occurs between the instant when a stored $\operatorname{Exp}(t, k)$ becomes allowed and the instant when the Exp-Delayer generates it. This assumption can be removed by using the following mechanism. Each event Exp is timestamped by the instant when the Timer-Handler generates it. When the Test-Controller receives an event (input or output) $x$ of the $\mathcal{I U T}$ and an event Exp $e$ from the Exp-Delayer, if $x$ precedes $e$ by a short delay then the Test-Controller determines the correct order of the two events from the timestamp of $e$. In the remaining of this article, for the sake of simplicity the use by the Test-Controller of such a mechanism is implicit. 


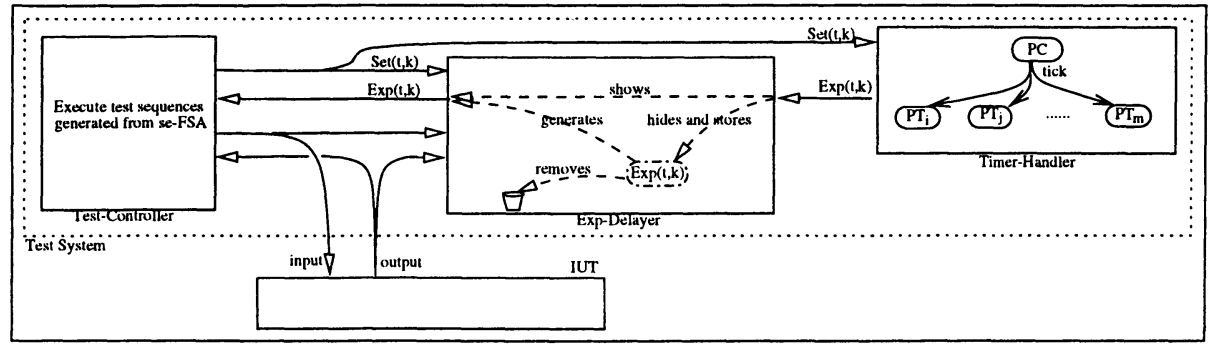

Figure 7. Structure of the test system

\subsection{Model of $\mathcal{I U T}$}

Since the specification of the $\mathcal{I U T}$ is initially described by a TA, we assume that the $\mathcal{I U T}$ may itself be described by a TA (possibly unknown). The proposed model of $\mathcal{I U T}$ is inspired from [6] and is represented in Fig. 8. The $\mathcal{I U T}$ consists of two parts :

A IUT-Controller which executes the TA modelling the $\mathcal{I U T}$

A Timer-Handler : (different than the Timer-Handler of the $\mathcal{T S}$ )

- a process $P C$ which is similar to the process $P C$ of $\mathcal{T S}$; and

- a process $P T_{i}$ is created with each ResetTimer $\left(t_{i}\right)$ which sets the value of $t_{i}$ to zero. When the timer handler receives PleaseValue $\left(t_{i}\right)$ then it immediately sends GetValue $\left(t_{i}, k\right)$ where $k$ is the current value of $t_{i}$.

With this model, we assume that the $P C$ of the $\mathcal{T S}$ and the $P C$ of the $\mathcal{I U T}$ are synchronized. In a first approach of synchronization, the two $P C$ s have a direct access to a single time source. In a more usual approach, each of the $P C$ s uses an internal hardware clock that gives an adequate approximation to the passage of time in the environment. With this approach, it is necessary to coordinate the two local clocks. There are many algorithms for doing this [16].

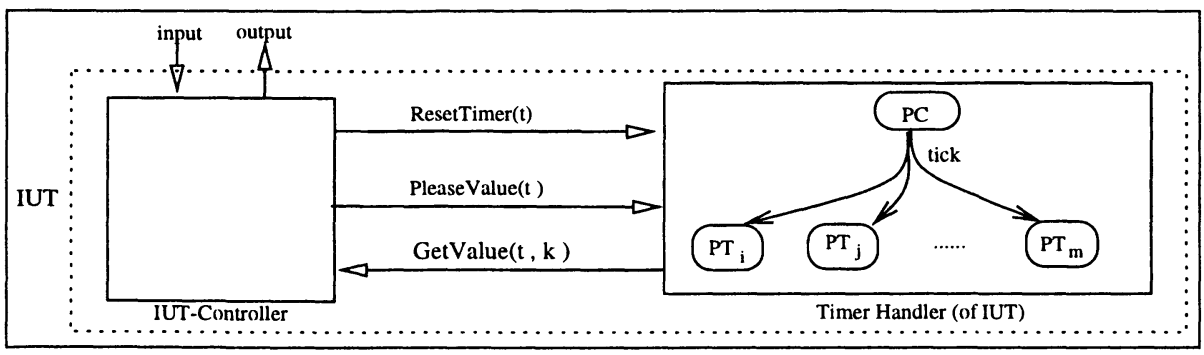

Figure 8. Model of $\mathcal{I U T}$ 


\section{TEST CASE GENERATION}

\subsection{Transforming se-FSA into io-FSA}

We intend to use a test generation method which is applicable to input/output FSAs (denoted io-FSA). For this reason, the se-FSA obtained by the algorithm of Sect. 4 needs to be transformed into a io-FSA. For the se-FSA of Fig. 4, after the removal of undesirable states and the transformation into a io-FSA, we obtain the io-FSA of Fig. 9.

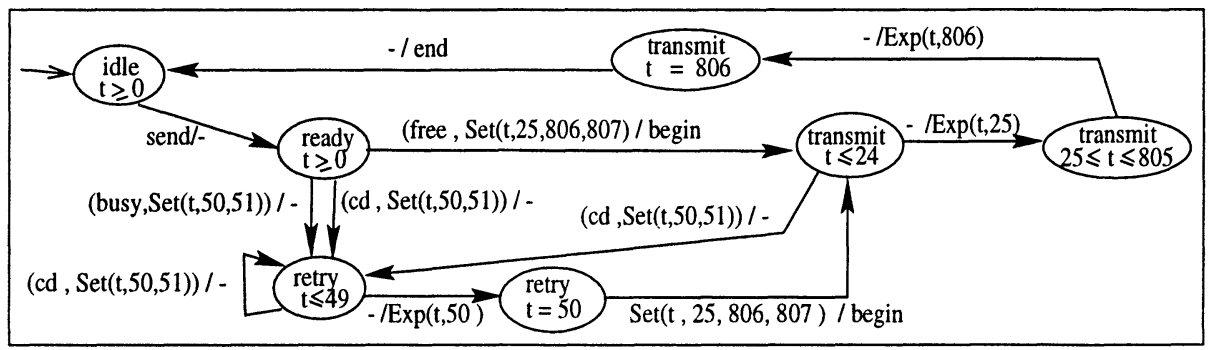

Figure 9. Input/output FSA obtained from the se-FSA of Fig. 4

\subsection{Use of Wp-Method}

For generating test sequences, we can use any software tool based on WpMethod [8]. As an example, we have used TAG [18] (Test Automatic Generation). First of all TAG is used to construct, for each state $q$ of the io-FSA : (where $q_{0}$ is the initial state of the io-FSA)

An Identification set $W_{q}$ which is a set of input sequences which can be used to identify $q$.

A Preamble $P_{q}$ which is an input sequence which brings the io-FSA from $q_{0}$ to $q$.

A Postamble $R_{q}$ which is an input sequence which brings the io-FSA from $q$ to $q_{0}$.

Then for each transition $\operatorname{Tr}:$ " $q \stackrel{\sigma / \rho}{\longrightarrow} r$ " of the io-FSA, TAG is used to construct a set of test cases, denoted TestCase ${ }_{T r}$, which allow to check Tr. Each test case is defined by : $P_{q}, \sigma, \operatorname{seq}\left(\in W_{r}\right)$ and $R_{s}$, where $s$ is the state reached after the execution of $s e q$ from $r$. Therefore, all test cases of TestCase ${ }_{T r}$ are differenciated by the input sequence of $W_{r}$ which is used to identify Tr. A test case is therefore executed into the following four steps :

1 Execute $P_{q}$ to bring the io-FSA from its initial state to State $q$;

2 Execute $\sigma$ and check whether the observed output(s) is(are) $\rho$;

3 Execute an input sequence $s e q$ of $W_{r}$ and check the observed output in order to identify the destination state of $\operatorname{Tr}$. Let $s$ be the state reached after the execution of seq.

4 Execute $R_{s}$ to bring the io-FSA from State $s$ to its initial state. 


\subsection{Test Hypotheses}

Correctness of the results obtained from TAG (or any tool based on WpMethod [8]) is guaranteed only if the following hypotheses hold.

Hypothesis 1 The TA describing a specification is deterministic, i.e., from any location of the TA we cannot have :

- several outgoing transitions labeled with the same input and the enabling conditions (EC) of which can be satisfied simultaneously;

- several outgoing transitions labeled by outputs and the EC of which can be satisfied simultaneously.

Hypothesis 2 The io-FSA derived from the TA describing the specification is deterministic, i.e., from any state of the io-FSA, we cannot have several transitions with the same (possibly empty) input.

The io-FSA must also satisfy the following other hypotheses :

1 It must contain no transition with an empty input. Since the io-FSA contains transitions "-/Exp", this hypothesis is never initially satisfied. A solution consists of replacing every "- $/ x$ " by " $w / x$ ", where $w$ is an internal action which models "waiting the event $x$ ". This solution can be applied to the example of Fig. 9.

2 It must be completely specified over the set of inputs; otherwise, a solution consists of completing it by adding a selfloop " $\sigma /-$ " in each state $q$ where an input $\sigma$ is not specified.

3 It must be minimal, i.e. it has no undistinguishable states. This hypothesis is always satisfied because the corresponding se-FSA generated by the algorithm of Sect 4 is minimal.

\subsection{Exp-Undeterministic io-FSAs}

Let $A$ and $B$ be a TA and the corresponding io-FSA, respectively. Hypothesis 2 is equivalent to Hypothesis 1 in the case where, to each location of $A$ corresponds a single sequence of "-/Exp" in $B$. As an example, the TA of Fig. 2 and its corresponding io-FSA of Fig. 9 are both deterministic. On the other hand, Hypothesis 2 is stronger than Hypothesis 1 in the case where, to a given location of $A$ correspond several sequences of "-/Exp" in $B$. In this case, we say that the io-FSA is Exp-Undeterministic. As an example, the TA of Fig. 5 is deterministic and its corresponding io-FSA (which can be obtained from Fig. 6.d) is Exp-Undeterministic. Therefore, TAG cannot be used for this io-FSA.

Here is an approach which allows to use TAG for Exp-Undeterministic ioFSAs. If $B$ is Exp-Undeterministic, then we generate several deterministic io-FSAs $B_{1}, B_{2}, \cdots$. The only difference between the $B_{i}$ s and $B$ is that, for 
each set of sequences of Exp of $B$ which correspond to the same location of $A$, each $B_{i}$ will have only a single sequence. For example, for the io-FSA corresponding to the se-FSA of Fig. 6.d, we obtain four io-FSAs containing one of the four sequences of Exp, respectively. TAG can then be used to generate test cases for each of them.

But if we consider test execution, a tester does not know in advance which sequence, among a set of sequences of Exp corresponding to the same location, will be executed. To deal with this problem, we propose that each test purpose be : To check any transition among a set of transitions which are reachable by paths : (1) with the same length; and (2) which diverge by transitions Exp. For the example in Fig. 6.d, the following three sets of transitions constitute three test purposes :

- the three outgoing transitions of State $L 2_{0}$;

- the five outgoing transitions of States $L 2_{1}$ and $L 2_{2}$;

- the two outgoing Exp of States $L 2_{3}$ and $L 2_{4}$.

\subsection{Procedure of Test Generation}

\begin{tabular}{|l|l|}
\hline Entry & TA \\
\hline Step 0 & $\begin{array}{l}\text { If Hypothesis 2 is not satisfied Then : terminate } \\
\text { Else }\end{array}$ \\
\hline Step 1 & Transform the TA into a se-FSA (see Sect. 4) \\
\hline Step 2 & Transform the se-FSA into a io-FSA (see Sect. 6.1) \\
\hline Step 3 & Remove empty inputs (see Point 1 in Sect. 6.3) \\
\hline Step 4 & Complete the io-FSA (see Point 2 in Sect. 6.3) \\
\hline Step 5 & $\begin{array}{l}\text { If the obtained io-FSA is Exp-Undeterministic Then : } \\
\text { Generate several deterministic io-FSAs } B_{1}, B_{2}, \cdots \text { (see Sect. 6.4) } \\
\text { End-If }\end{array}$ \\
\hline Step 6 & $\begin{array}{l}\text { For each deterministic io-FSA : } \\
\text { Compute Identification Sets, Preambles, Postambles, and Test cases } \\
\text { Remove test cases containing selfloops which have been added at Step 4 } \\
\text { End-For }\end{array}$ \\
\hline & End-If \\
\hline
\end{tabular}

\subsection{Example}

Let us consider the specification of Fig. 2. Hypothesis 2 is satisfied and after Step 2 of the test procedure, we obtain the io-FSA of Fig. 9. After the application of the whole test procedure, we obtain the following identification sets, preambles and postambles.

\section{Identification sets :}

$$
\begin{aligned}
& W_{\langle\text {idle }, t \geq 0\rangle}=W_{\langle\text {retry }, t=50\rangle}=\{w \cdot \text { free_Set }(t, 25,806,807) \cdot \operatorname{Set}(t, 25,806,807)\} \\
& W_{\langle\text {ready }, t \geq 0\rangle}=\{w \cdot \text { free_Set }(t, 25,806,807)\} \\
& W_{\langle\text {transmit }, t \leq 24\rangle}=W_{\langle\text {transmit }, 25 \leq t \leq 805\rangle}=W_{\langle\text {transmit }, t=806\rangle}=W_{\langle\text {retry }, t \leq 49\rangle}=\{w\}
\end{aligned}
$$

Preambles :

$$
P_{\langle i d l e, t \geq 0\rangle}=\epsilon \text { (i.e., empty); } P_{\langle\text {ready }, t \geq 0\rangle}=\text { send; }
$$




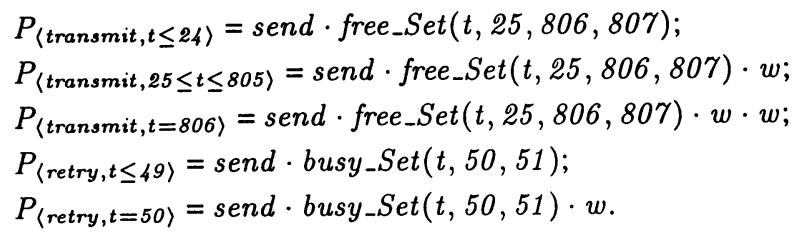

Postamble :

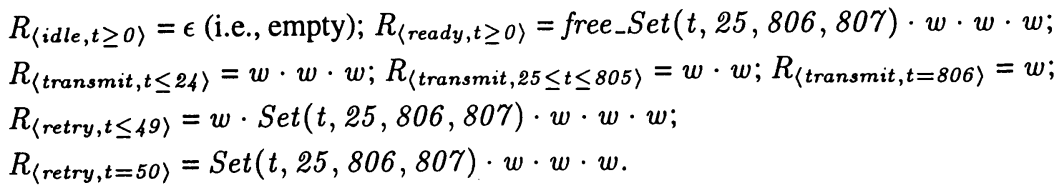

Since here each $W_{q}$ is a singleton, a single test case is sufficient for checking a transition. Let us mention twelve specific test cases which allow to check each of the twelve transitions of the io-FSA. For each transition $\operatorname{Tr}:$ : $q \stackrel{\sigma / \rho}{\longrightarrow} r$ ", the corresponding test case is therefore defined by : $P_{q}, \sigma, W_{r}$ and $R_{s}$, where $s$ is the state reached after the execution of $W_{r}$ from $r$ (see Sect. 6.2). For lack of space, we do not present here the twelve test cases, but note that they can be easily deduced because all parameters they depend on are presented.

\section{CONCLUSION AND FUTURE WORK}

\subsection{Contributions}

This study deals with testing a protocol entity with timing constraints. If we consider previous works in this area, we think that those presented in $[17,6]$ are among the most interesting. Contrary to the latters who use a continuous time, in the present study we use a discrete time. In comparison with $[17,6]$, our main contribution is that we propose a test generation method and a test architecture which avoid state explosion. For that purpose, we have used two special events called Set and Exp which are generated by the test system and which represent the setting and expiration of timers, respectively.

\subsection{Future Work}

In the near future, we intend to investigate the following issues :

- To determine several rules which can be used to remove impossible sequences of Exp. In the present study, we have used a single rule (see Point 3 in Step 3 of Sect. 4.2).

- To study certain problems of test execution. Several aspects need to be considered such as :

1 When a io-FSA $B$ is Exp-Undeterministic, we propose in Sect. 6.4 to replace $B$ by several deterministic io-FSAs $B_{1}, B_{2}, \cdots$ and then to generate test cases from these deterministic io-FSAs. But during 
a test execution, the tester does not know in advance which sequence of $E x p$ will be executed. In Sect. 6.4 we propose an idea to deal with this problem, but we intend to study more thoroughly this problem in order to propose a complete procedure of test execution.

2 If certain impossible sequences of Exp remain in the io-FSA, the test generation procedure will generate test cases for checking impossible (i.e., unreachable) transitions. Therefore during a test execution, the tester will try to check unreachable transitions. An idea to deal with this problem is : if after a given number $N$ of attempts the tester does not succeed to execute a transition $T r$, then it may consider that Tr is unreachable. The question which arises is : how to select $N$ ? We intend to study more thoroughly this problem.

- To study the test of distributed systems.

- To apply and possibly adapt the proposed method for testing multimedia applications with timing constraints.

\section{References}

[1] R. Alur, C. Courcoubetis, D. Dill, and H. Wong-Toi. Minimization of timed transitions systems. In CONCUR, pages 341-354. Springer-Verlag LNCS 630, 1992.

[2] R. Alur and D. Dill. A theory of timed automata. Theoretical Computer Science, 126:183235, 1994.

[3] D. Clarke and I. Lee. Automatic generation of tests for timing constraints from requirements. In Third International Workshop on Object-Oriented Real-Time Dependable Systems, Newport Beach, California, February 1997.

[4] D. L. Dill. Timing assumptions and verification of finite-state concurrent systems. In Automatic Verification Methods for Finite State Systems, pages 197-212. Springer-Verlag LNCS 407, 1989.

[5] A. En-Nouaary, R. Dssouli, and A. Elqortobi. Génération de tests temporisés. In 6th Colloque Francophone de l'Ingénierie des Protocoles. HERMES, 1997.

[6] A. En-Nouaary, R. Dssouli, F. Khendek, and A. Elqortobi. Timed test generation based on state characterization technique. In 19th IEEE Real-Time Systems Symposium (RTSS), Madrid, Spain, December 1998.

[7] A. En-Nouaary, F. Khendek, and R. Dssouli. Fault coverage in testing real-time systems. In 6th Intern. Conf. on Real-Time Computing Systems and Applications (RTCSA), HongKong, December 1999.

[8] S. Fujiwara, G. v. Bochmann, F. Khendek, M. AAmalou, and A. Ghedamsi. Test selection based on finite state models. IEEE Transactions on Software Engineering, 17(6):591-603, 1991.

[9] A. Khoumsi, M. Akalay, A. En-Nouaary, R. Dssouli, and L. Granger. An approach for testing real time protocol entities. Technical Report 1173, Université de Montréal. Département IRO, Montréal, Québec, Canada, May 2000.

[10] A. Khoumsi and K. Saleh. Two formal methods for the synthesis of discrete event systems. Computer Networks and ISDN, 29:759-780, 1997. 
[11] M. Lawford and W.M. Wonham. Equivalence preserving transformations of timed transitions models. IEEE Transactions on Automatic Control, 40:1167-1179, July 1995.

[12] F. Liu. Test generation based on an FSM model with timers and counters. Master's thesis, University of Montreal, Department IRO, 1993.

[13] G. Luo, R. Dssouli, G.v. Bochmann, P. Venkataram, and A. Ghedamsi. Test generation with respect to distributed interfaces. Computer Standards and Interfaces, 16:119-132, 1994.

[14] D. Mandrioli, S. Morasca, and A. Morzenti. Generating test cases for real-time systems from logic specifications. ACM Transactions on Computer Systems, 13(4):365-398, November 1995.

[15] J.S. Ostroff and W.M. Wonham. A framework for real-time discrete event control. IEEE Transactions on Automatic Control, 35(4):386-397, 1990.

[16] P. Ramanathan and K.G. Shin. Fault tolerant clock synchronization in distributed systems. IEEE Computer, 23:33-42, October 1990.

[17] J. Springintveld, F. Vaadranger, and P. Dargenio. Testing timed automata. Technical Report CTIT97-17, University of Twente, Amsterdam, The Netherlands, 1997.

[18] Q. M. Tan, A. Petrenko, and G. v. Bochmann. A test generation tool for specifications in the form of state machines. In International Communications Conference (ICC), pages 225-229, Dallas, Texas, June 1996.

[19] A. Tanenbaum. Computer Networks. Prentice-Hall (3rd edition), 1996. 